\title{
Magnetic Flux Leakage Sensing-Based Steel Cable NDE Technique
}

\author{
Seunghee Park, ${ }^{1}$ Ju-Won Kim, ${ }^{2}$ Changgil Lee, ${ }^{1}$ and Jong-Jae Lee ${ }^{3}$ \\ ${ }^{1}$ School of Civil, Architectural, and Environmental Engineering, Sungkyunkwan University, Suwon, \\ Gyeonggi 440-746, Republic of Korea \\ ${ }^{2}$ Department of u-City Design and Engineering, Sungkyunkwan University, Suwon, Gyeonggi 440-746, Republic of Korea \\ ${ }^{3}$ Department of Civil and Environmental Engineering, Sejong University, Seoul 143-747, Republic of Korea
}

Correspondence should be addressed to Seunghee Park; shparkpc@skku.edu

Received 11 December 2012; Accepted 13 April 2013; Published 8 May 2014

Academic Editor: Gyuhae Park

Copyright (C) 2014 Seunghee Park et al. This is an open access article distributed under the Creative Commons Attribution License, which permits unrestricted use, distribution, and reproduction in any medium, provided the original work is properly cited.

\begin{abstract}
Nondestructive evaluation (NDE) of steel cables in long span bridges is necessary to prevent structural failure. Thus, an automated cable monitoring system is proposed that uses a suitable NDE technique and a cable-climbing robot. A magnetic flux leakage(MFL-) based inspection system was applied to monitor the condition of cables. This inspection system measures magnetic flux to detect the local faults (LF) of steel cable. To verify the feasibility of the proposed damage detection technique, an 8-channel MFL sensor head prototype was designed and fabricated. A steel cable bunch specimen with several types of damage was fabricated and scanned by the MFL sensor head to measure the magnetic flux density of the specimen. To interpret the condition of the steel cable, magnetic flux signals were used to determine the locations of the flaws and the levels of damage. Measured signals from the damaged specimen were compared with thresholds that were set for objective decision-making. In addition, the measured magnetic flux signals were visualized as a 3D MFL map for intuitive cable monitoring. Finally, the results were compared with information on actual inflicted damages, to confirm the accuracy and effectiveness of the proposed cable monitoring method.
\end{abstract}

\section{Introduction}

Recently, there have been increasing demands on structural health monitoring (SHM) and nondestructive testing (NDT) in the fields of civil, mechanical, and aerospace engineering. Especially, local monitoring methodologies for specific critical members have been studied to overcome the limitation of global monitoring techniques for whole structures [1-4]. Steel cables in long span bridges are also critical members that suspend almost all of the dead load of the structure. However, cross-sectional damage can occur in a steel cable due to corrosion and fracture, which can lead to stress concentrations. Cross-sectional damage can be a direct cause of structural failure. Therefore, nondestructive evaluation (NDE) is necessary to detect the initial stages of crosssectional damage in a cable. However, it is difficult to monitor the condition of most cables, as the damage can be invisible and inaccessibly located. To overcome these drawbacks, we propose an automated cable monitoring system, which uses a suitable NDE technique and a cable-climbing robot that can approach the damaged point, which is shown in Figure 1.

Meanwhile, NDE techniques available for incorporation into cable-climbing robots have been widely researched. In this study, a magnetic sensor for the detection of crosssectional damage was applied. Magnetic sensors are widely used to monitor structures, including aircrafts and ships, due to their excellent reliability and reproducibility. Various kinds of magnetic sensors exist, and optimal magnetic properties can be utilized according to the kind of target structure [5-9].

In this study, an MFL sensor was applied to detect the local fault (LF) damage of steel cables, by capturing the magnetic flux leakage. The magnetic flux leakage method is most suitable for continuous structures that have constant cross-sections, such as cables and pipes, and has been applied for the inspection of steel cables in the mining industry, for ski lifts, elevators, and for other applications [10-15]. However, most MFL devices are fixed systems, which cannot be used for steel cables in bridges. In addition, the measured signals 


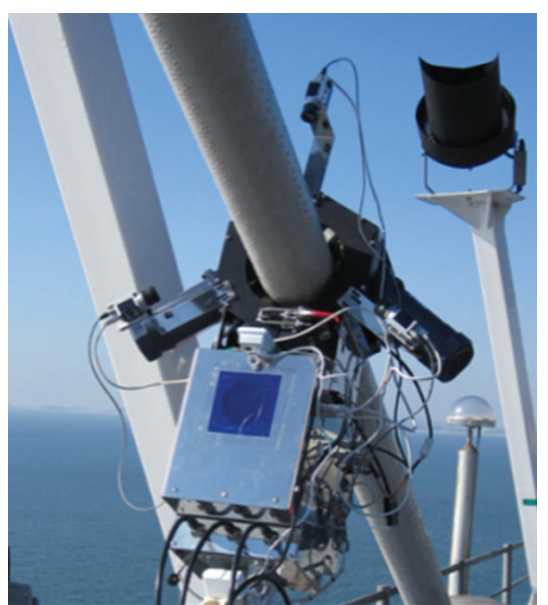

(a)

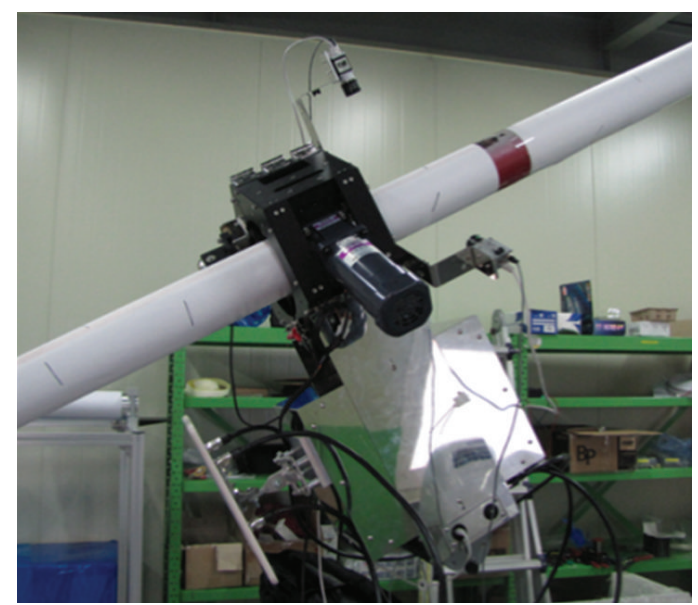

(b)

FIGURE 1: The cable-climbing robot with suitable NDE equipment.

from MFL devices require analysis by experts to determine damage. To overcome these limitations, an advanced MFLbased damage detection technique has been developed that utilizes thresholds created from statistical methods used for objective decision-making.

To verify the feasibility of the proposed damage detection technique, an 8-channel MFL sensor prototype was designed and fabricated. A steel cable bunch specimen was also fabricated to perform the experiment, in which cross-sectional damages were formed step-by-step, by making cuts in the cable. The sensor was used to measure magnetic flux at each damage condition. Measured signals from the damaged specimen were compared with the set thresholds. Finally, the measured magnetic flux signal was visualized as a 3D MFL map, for intuitive cable monitoring.

\section{Theoretical Backgrounds}

2.1. Principle of Magnetic Flux Leakage. A steel specimen that is magnetized has a magnetic field in and around itself, and any place where a magnetic line of force exits or enters the

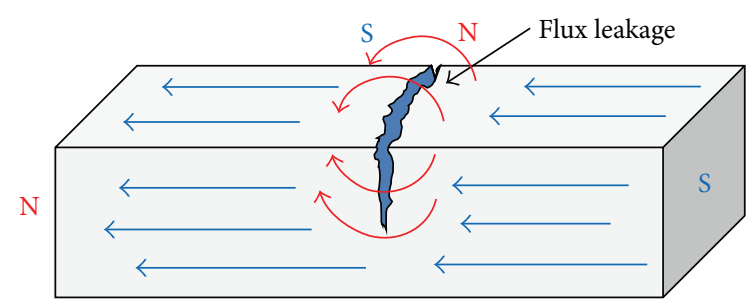

FIGURE 2: Principle of magnetic flux leakage.

specimen is called a pole. A magnet that is cracked, but not broken completely in two, forms a north and south pole at each edge of the crack, as shown in Figure 2. The magnetic field exits in the north pole and reenters the south pole. The magnetic field spreads out when it encounters the small air gap created by the crack, because the air cannot support as much magnetic field per unit volume as the magnet can. When the field spreads out, it appears to leak out of the material and is thus called a flux leakage field.

2.2. Magnetic Flux Leakage-Based Local Fault Damage Detection Technique. A strong permanent magnet or an electromagnet is used to establish a magnetic flux in the material to be inspected. When there is no defect, the flux in the metal remains uniform, as illustrated in Figure 3(a). In contrast, Figure 3(b) illustrates the flux leakage that occurs when there is LF damage, due to broken wire or wear. The flux leaks out of the metal near the defect. Sensors that can detect this flux leakage are placed between the poles of the magnet and they generate an electric signal that is proportional to the magnetic flux leakage [16].

In this study, Hall sensors were used to capture the MFL. The Hall sensor operates based on the Hall effect and is illustrated in Figure 4. When a magnetic field is applied to a plate, an electron moving through a magnetic field experiences a force, known as the Lorentz force, that is perpendicular both to the direction of motion and to the direction of the field. It is the response to this force that creates the Hall voltage $[17,18]$. This Hall voltage can be measured using a DAQ system and can be used to examine the condition of target structure.

\subsection{Establishment of a Threshold Level Using GEV Distribu-} tion. After measuring the magnetic flux, it is necessary to determine an appropriate threshold for the measured output voltage that will distinguish between an intact and damaged condition. In this study, a $99.99 \%$ confidence level threshold of the intact condition was set, using the generalized extreme value (GEV) distribution. By the extreme value theorem, the GEV distribution is the limit distribution of the properly normalized maxima of a sequence of independent and identically distributed random variables. Because of this, the GEV distribution is used as an approximation, to model the maxima of long (finite) sequences of random variables. 


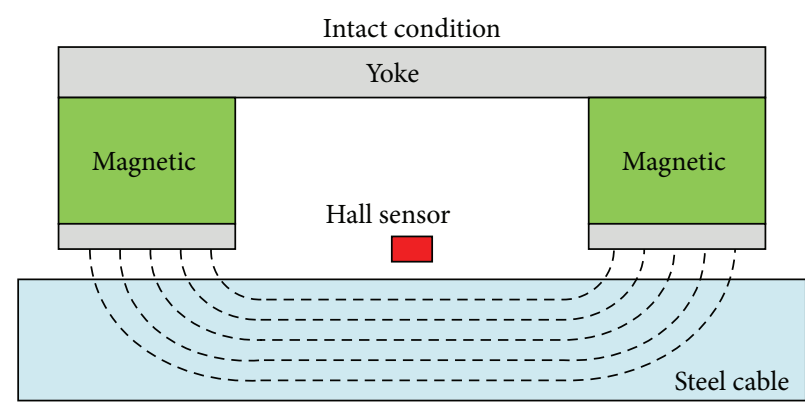

(a) Intact condition

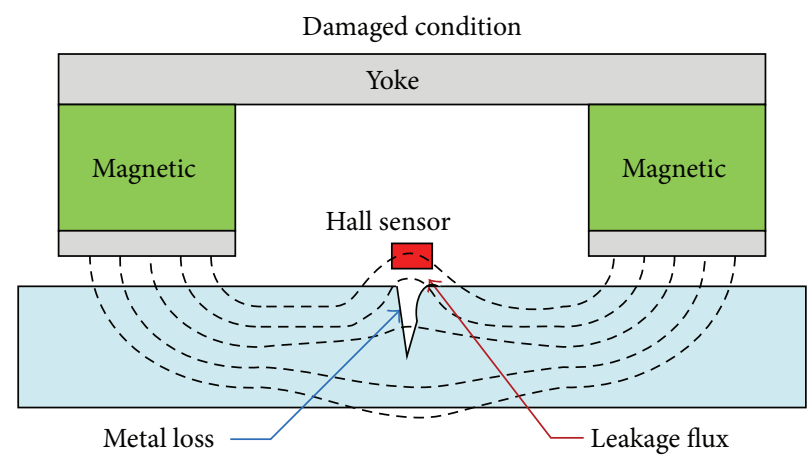

(b) Damaged condition

FIgURE 3: The concept of MFL-based LF damage detection technique.

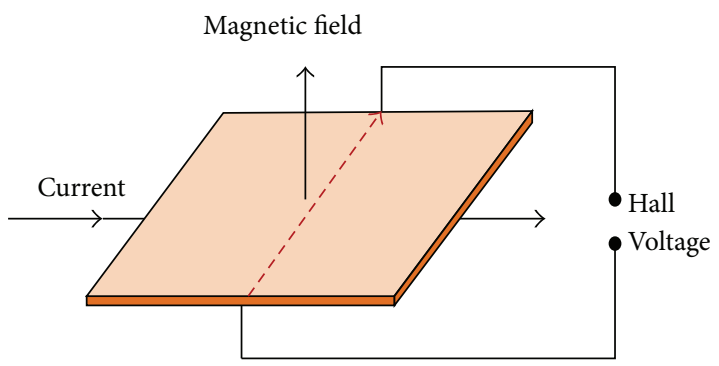

Figure 4: Principle of the Hall effect.

The generalized extreme value distribution has a cumulative distribution function, as shown in

$$
F(x ; \mu, \sigma, \xi)=\exp \left\{-\left[1+\xi\left(\frac{x-\mu}{\sigma}\right)\right]^{-1 / \xi}\right\}
$$

for $1+\xi(x-\mu) / \sigma>0$, where $x$ is the upper end-point, $\mu \in \mathbb{R}$ the location parameter, $\sigma>0$ the scale parameter, and $\xi \in \mathbb{R}$ the shape parameter [19].

\section{Experimental Study}

3.1. Design and Fabrication of MFL Sensor Head Prototype. An MFL sensor head prototype was fabricated, as shown in Figure 5, to perform the experimental study to verify the proposed cable NDE method. The sensor head was composed of a magnetization component and a signal measurement component, which were packed into an aluminum case of $18 \mathrm{~cm}$ width, $18 \mathrm{~cm}$ height, and $30 \mathrm{~cm}$ length. Additionally, the components could be detached conveniently, by making a system that could be readily opened.

First, the magnetization component, which creates the magnetic field for magnetizing a portion of the steel cable specimen, is a pair of yokes, which consist of 2 high strength $\mathrm{Nd}-\mathrm{Fe}-\mathrm{B}$ permanent magnets (neodymium 35) and a plate of carbon steel, as in Figure 6(a). The permanent magnet has the advantage of not requiring any power for operation and allows for a lighter system than if an electromagnetic magnet was to be used.

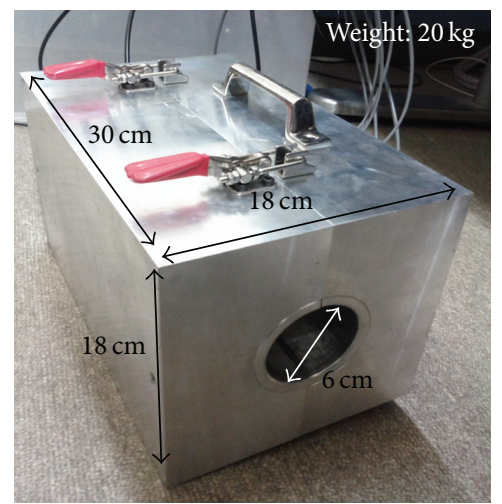

(a)

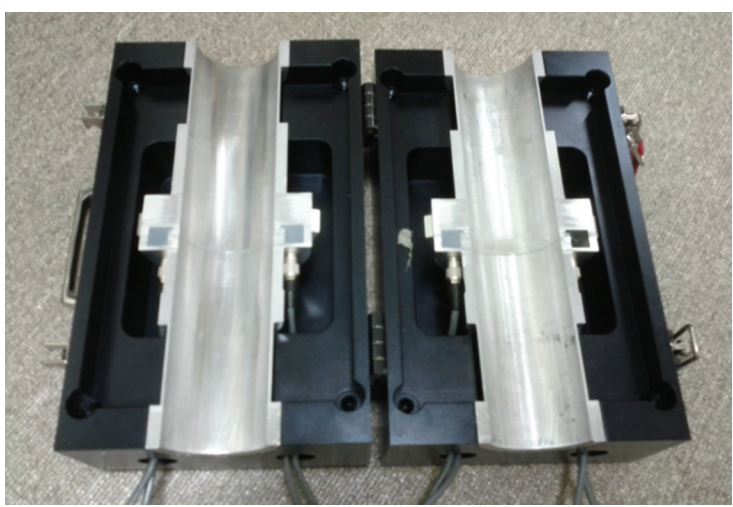

(b)

Figure 5: The fabricated MFL sensor head prototype.

The signal measurement component is composed of 8 channels of Hall sensors, which are circumferentially arranged at regular intervals around a circular configuration as shown in Figure 6(b). Each of the 8 arranged sensors converts the MFL signal to a voltage signal, which is transmitted to a DAQ system. The inner diameter of the sensor head is $60 \mathrm{~mm}$, to accommodate steel cable of the same dimension.

3.2. Experimental Setup and Procedure. A series of experimental studies were carried out to examine the capabilities of the detection technique. A steel cable specimen of $60 \mathrm{~mm}$ 
TABLE 1: Scenario of LF damage at specimen.

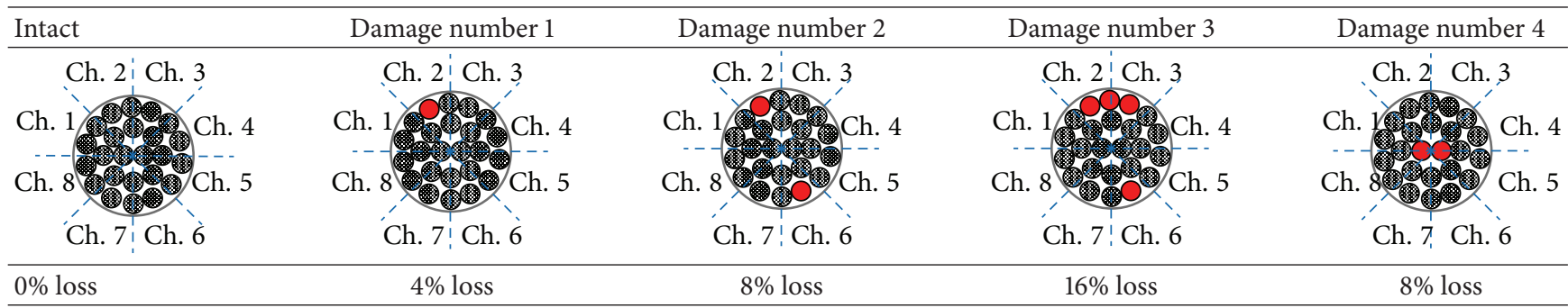

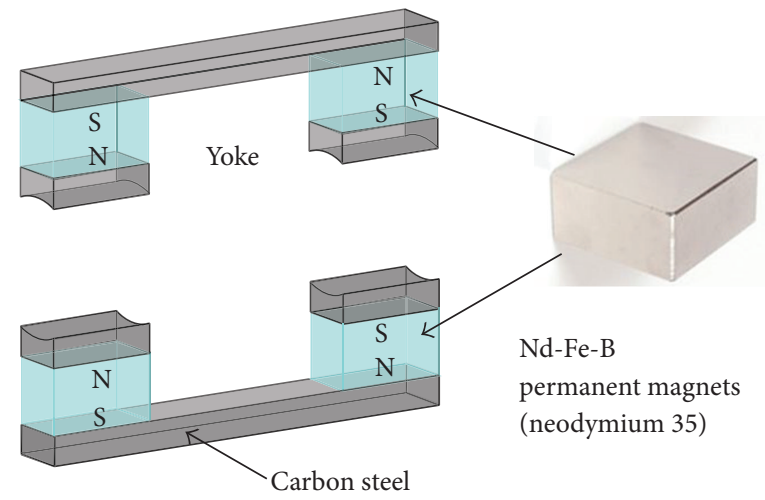

(a) Magnetization part

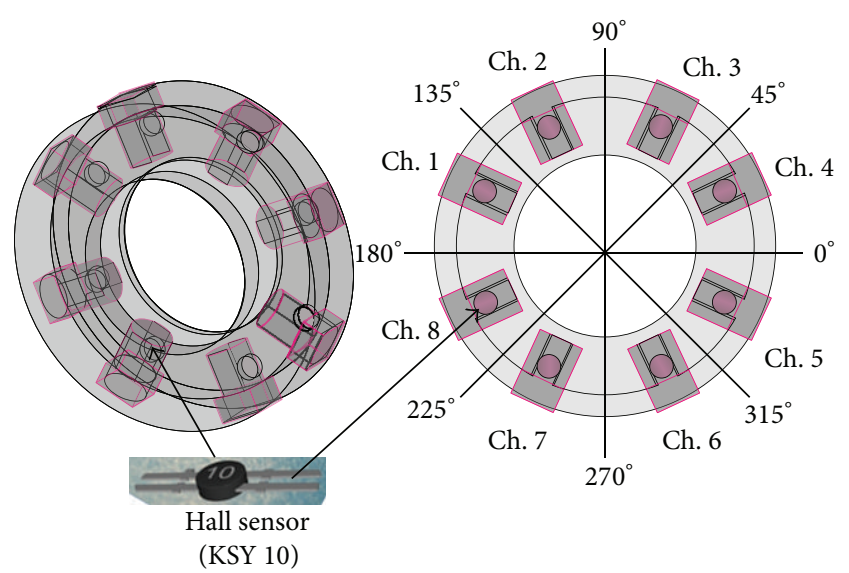

(b) Sensing part

FIgURE 6: Design of the MFL sensor head.

diameter and $1700 \mathrm{~mm}$ length was fabricated for the experiment, by filling 25 strands of steel cable in a pipe of $60 \mathrm{~mm}$ external diameter. Each strand of cable was stranded from $7 * 19$ element wires and was $10 \mathrm{~mm}$ in diameter, as shown in Figure 7.

Thereafter, 4 levels of LF damage were formed step-bystep at the center of the steel cable specimen, as shown in Table 1. In Table 1, a red dot means a fully cut damage of the strand which is specified as a $4 \%(1 / 25)$ cross-sectional loss of cable specimen. First, a cable located near sensing channel 2 was cut (damage level 1). Second, for damage level 2, a cable located in the plane of symmetry with damage level 1 was additionally cut (near sensing channel 6). At level 3, the cut

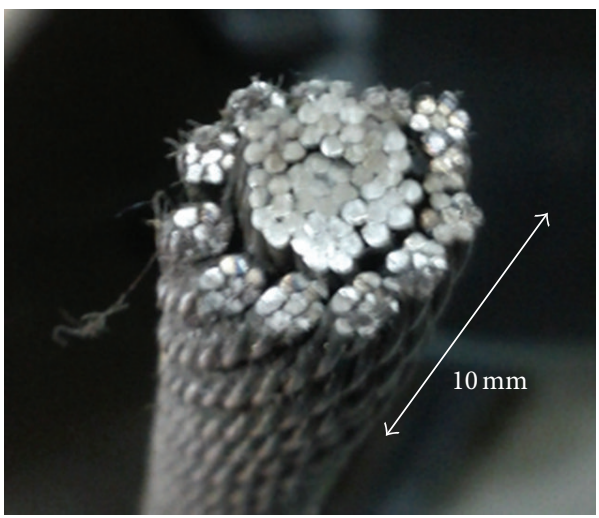

(a)

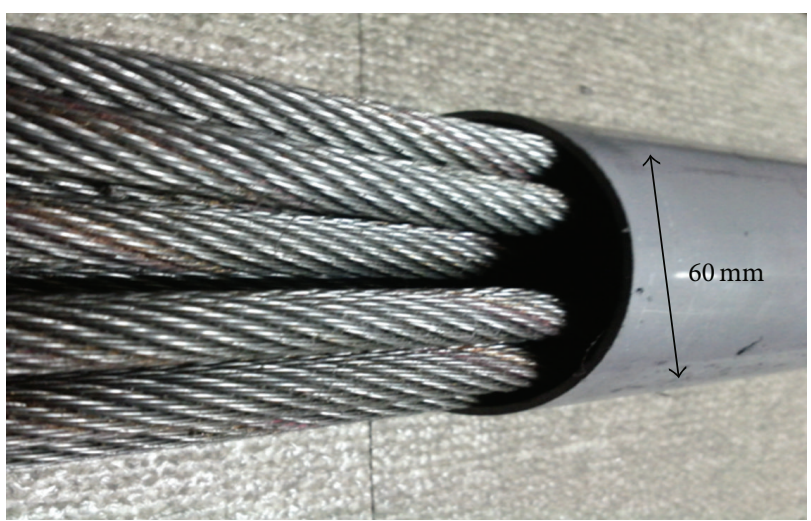

(b)

Figure 7: Specifications of the steel cable specimen.

damage was extended by cutting 2 more element cables in a clockwise direction as in Table 1. Finally, at damage level 4, 2 cables located at the center of the cross-section were cut, to form inner damage.

Using the sensor head, MFL signals were measured from only the center $1 \mathrm{~m}$ section of the damaged specimen for each damage level condition, as shown in Figure 8. The moving speed of the sensor head was $1 \mathrm{~m} / \mathrm{sec}$, and the sampling rate was $1 \mathrm{kHz}$. Therefore, the displacement resolution of this setup is $1 \mathrm{~mm}$.

Next, a virtual $5 \mathrm{~m}$ length of steel cable was rendered, by combining the 5-measurement sections of each damage condition (from intact to damage level number 4), as shown in Figure 9. 


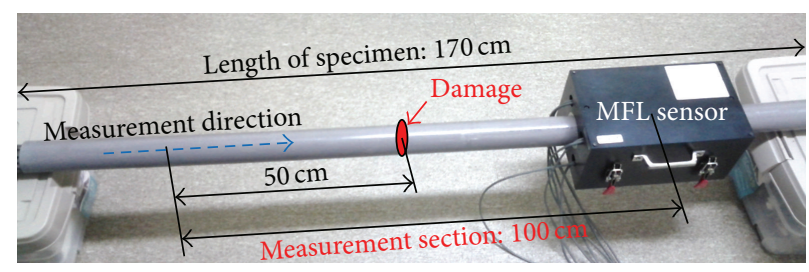

Figure 8: Test setup and measurement.

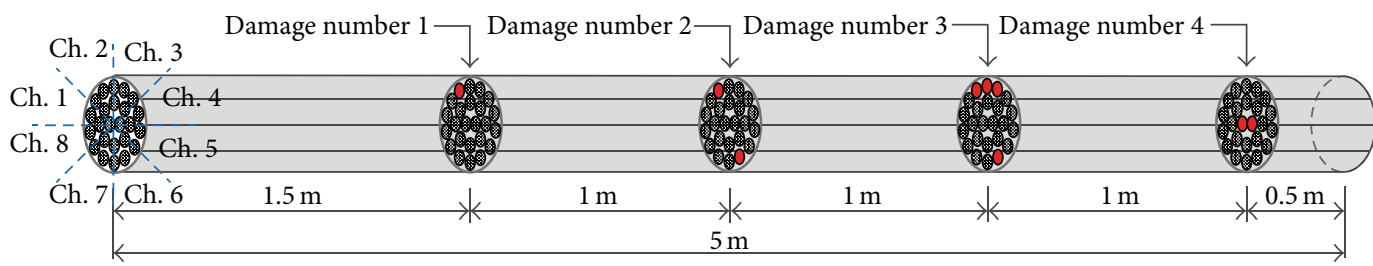

Figure 9: Virtual combined steel cable specimen.
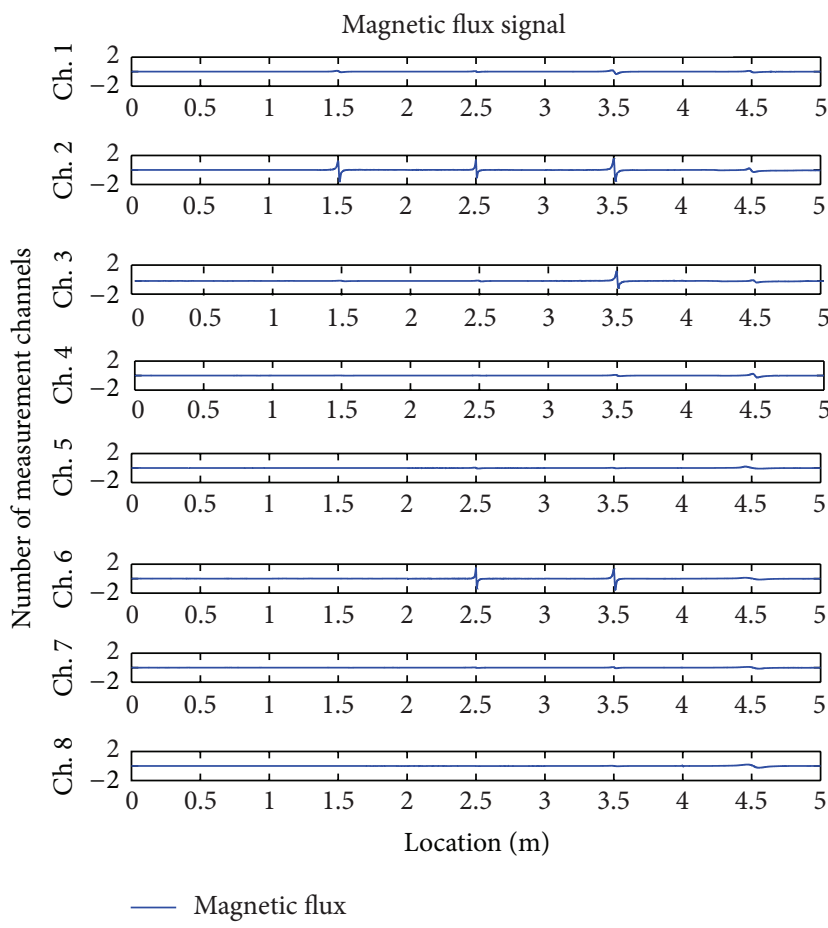

FIGURE 10: The raw magnetic flux signals from each channel.

Signal processing techniques, such as low-pass filtering and offset correction, were carried out to improve the resolution of the test results.

\subsection{Experimental Results}

3.3.1. Results of LF Damage Detection Based on MFL Method. The voltage signals that were measured from each channel of Hall sensors are displayed in Figure 10.

This figure shows symmetrical magnetic flux leakage signals measured at sensing channel 2 at the $1.5 \mathrm{~m}$ point along the length of the specimen and at channels 2 and 6 at the $2.5 \mathrm{~m}$ point. These MFL signals are consistent with the location of the actual local fault damage formed in the specimen. The flux leakage was captured only at sensing channels adjacent to the damages. At the $3.5 \mathrm{~m}$ point, the flux leakage signals were measured at sensing channels 2,3 , and 6 , and faint flux leakage signals were also measured at sensing channels 1 and 4. This shows that when the damage is greater, flux leakage can be measured in neighboring channels. In addition, small FL signals were captured from every channel at the $4.5 \mathrm{~m}$ point (where the cut damages are located at a similar distance from all sensing channels). These facts demonstrate that the MFL sensor method can detect LF damages, and sensing sensitivity is dependent on the distance between the damage and the Hall sensor. Additionally, the circumferential location of the damage can be judged, by arranging the sensors in an array.

A threshold that distinguishes between intact and damaged conditions was set, to determine the damage objectively. Original MFL signals were converted to absolute values so that only magnitudes would be considered in the thresholds. In this study, a 99.99\% confidence level threshold of the intact condition was set, using the generalized extreme value (GEV) distribution. The calculated threshold value is $0.1358 \mathrm{~V}$, which is shown with the converted MFL signal in Figure 11.

The converted flux leakage signals exceeded the threshold at sensing channel 2 at the $1.5 \mathrm{~m}$ point, channels 2 and 6 at the $2.5 \mathrm{~m}$ point, and channels 1,2, 3, and 6 at the $3.5 \mathrm{~m}$ point. At the $4.5 \mathrm{~m}$ point, the signals from almost all channels exceeded the threshold by a narrow margin. These measurements coincide with the actual damage locations and show that customized thresholds can be used as criteria to check whether or not damage has occurred.

3.3.2. Visualization of Magnetic Flux. The MFL signal was expressed in a 3-dimensional graph, as shown in Figure 12.

The $x$-axis is the distance along the specimen, the $y$ axis is the measurement channel number that represents the position of the sensor in the circumferential direction, and the amplitude of the magnetic flux is displayed on the $z$-axis and is color coded. Interpolation was performed to overcome the limitations of the limited number of sensing channels 

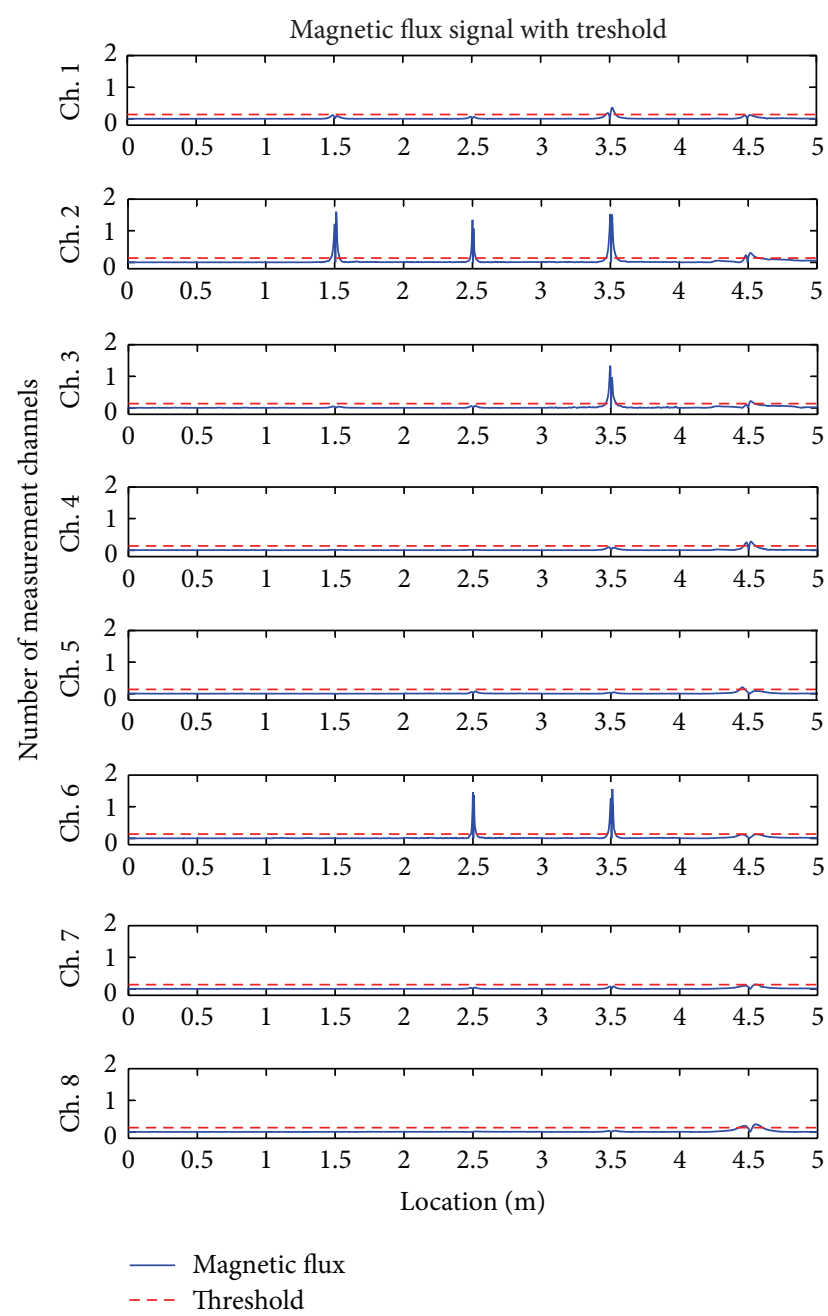

FIGURE 11: The converted magnetic flux signals with threshold.

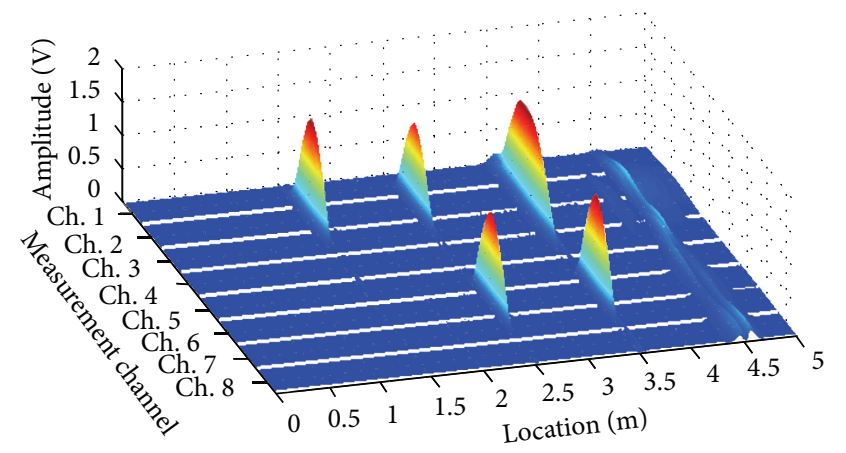

Figure 12: 3D magnetic flux graph.

for the smooth shape of the graph. In this figure, the large peaks that describe the flux leakage are clearly distinguishable from the intact signal, and they lay out the the size and location of the LF damages efficiently. However, small leakage was not distinguishable, due to noise in the signal. Thus, a threshold surface (shown in black) was added on the MFL graph, as shown in Figure 13. This precisely distinguishes the LF damaged areas from noise signals and the intact condition.

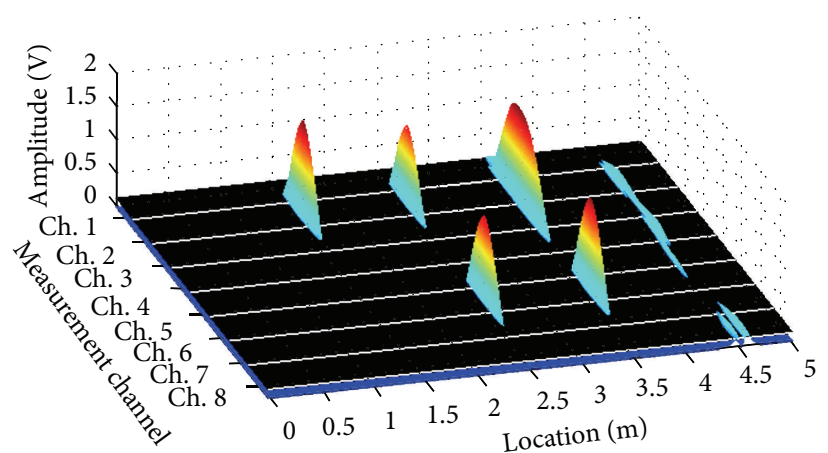

FIGURE 13: 3D magnetic flux graph with threshold surface.

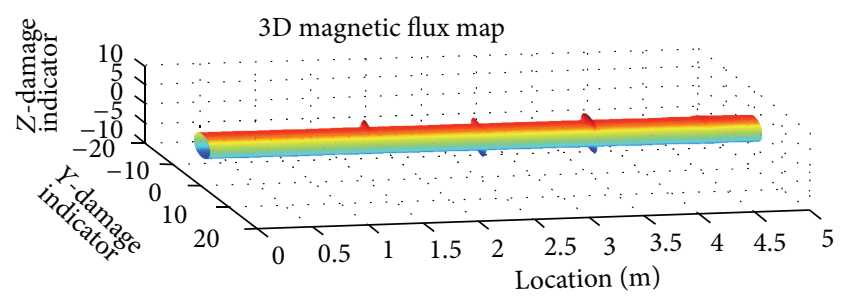

FIgURE 14: Visualized 3D magnetic flux map.

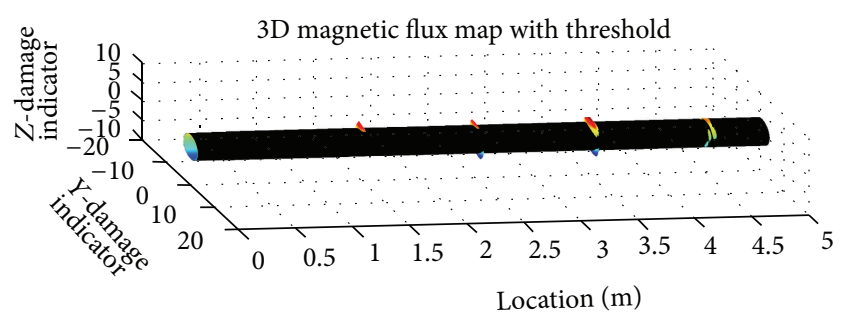

Figure 15: Visualized 3D magnetic flux map with threshold cover.

Finally, the MFL signals were visualized, by mapping onto a shape that is similar to the cable. For visualization, $y$ and $z$-dimensional damage indicators were calculated from converted magnetic flux values, using

$$
\begin{aligned}
& Y_{\mathrm{DI}}=\left(\mathrm{MF}_{c}+r\right) \times \cos \theta, \\
& Z_{\mathrm{DI}}=\left(\mathrm{MF}_{c}+r\right) \times \sin \theta,
\end{aligned}
$$

where $Y_{\mathrm{DI}}$ and $Z_{\mathrm{DI}}$ are the $y$ - and $z$-dimenional damage indicator, $\mathrm{MF}_{c}$ is the magnetic flux of cable, $r$ is a radius for virtual cable visualization, and $\theta$ is the circumferential angle of the sensor.

These calculated damage indicators were plotted on a $3 \mathrm{D}$ graph with a distance indicator, as shown in Figure 14.

This 3D magnetic flux map represents the damage location information in the longitudinal and circumferential directions and shows the level of damage at a glance, from any angle.

In addition, the $3 \mathrm{D}$ magnetic flux map was wrapped with a cylindrical threshold, in a manner similar to Figure 13, as shown in Figure 15. 


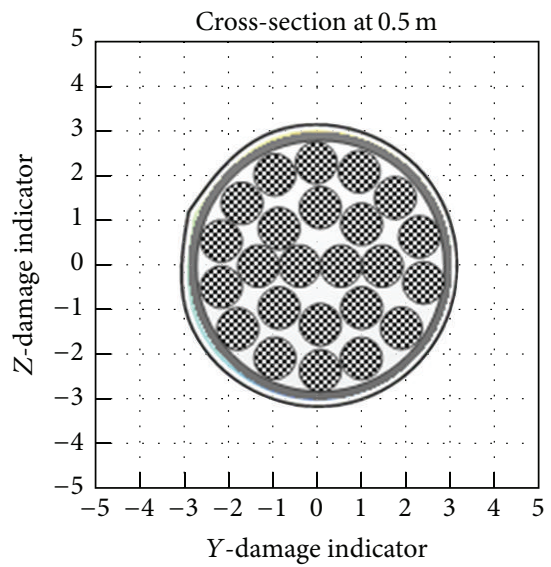

(a)

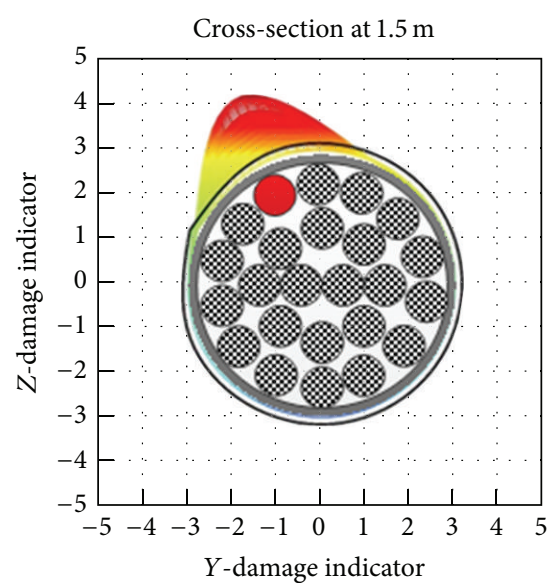

(b)

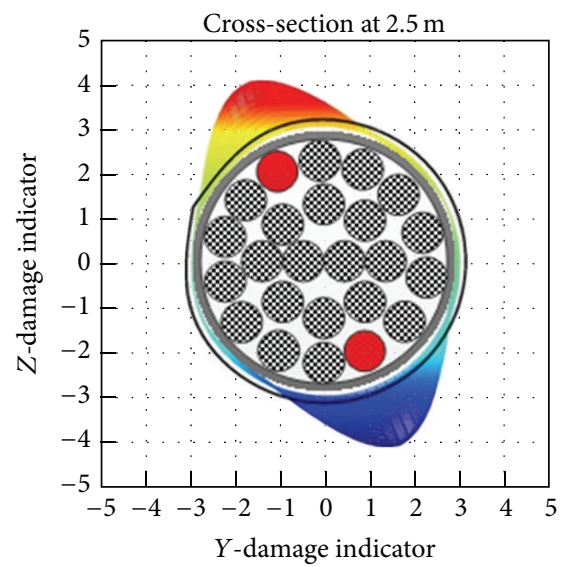

(c)

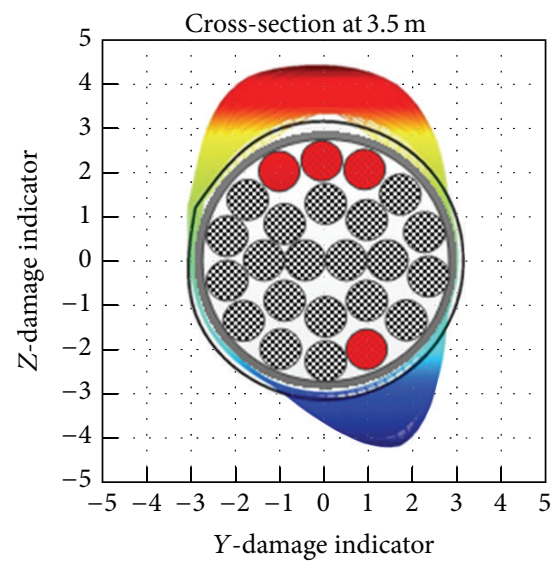

(d)

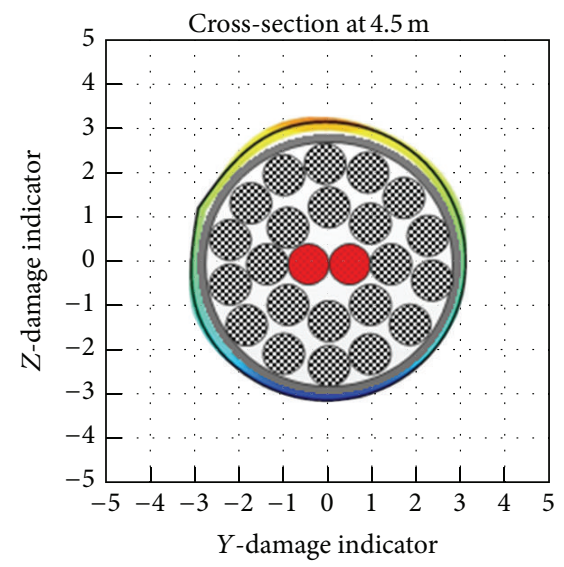

(e)

FIGURE 16: Visualized cross-sections with threshold cover.

This figure shows the damage parts highlighted by the threshold covering process. In addition, the circumferential direction and size of damage can be detected more efficiently by using cross-sections of the 3D MFL map, as shown in Figure 16.

Figure 16 shows visualized cross-sections with a threshold cover. Through this visualization work, it is expected that the damage in a cable can be detected by anyone, even by those with no professional experience with cable NDE.

\section{Conclusions}

An MFL sensor-based damage detection technique for the health monitoring of steel cables was proposed in this study. Fabrication of an MFL sensor head and a series of experimental studies and damage visualization were performed to verify the feasibility of the proposed technique, which was confirmed via the following observations.

(1) Magnetic flux leakage was detected at the locations of actual LF damage.

(2) The sensing sensitivity is dependent on the distance between the damage and the Hall sensors.
(3) The circumferential location of the damage can be judged by setting the sensors in an array.

(4) Flux leakage signals exceeded the thresholds based on a GEV distribution at the actual damage point.

(5) MFL signals were represented in a 3-dimensional graph that presents the size and location of LF damage at a glance.

(6) The MFL signals were represented with visualizations of the thresholds, by mapping the $3 \mathrm{D}$ graph onto a shape similar to the cable shape, which could efficiently provide information about the damage in the cable, from many angles.

Overall, these results demonstrated that the proposed steel cable monitoring technique using MFL sensors can be used to effectively detect local fault damage. In near future, this MFL- based cable NDE technique will be incorporated on cable-climbing robot to configure the automated cable monitoring system. In addition, it is expected that the proposed NDE technique can be utilized as an advanced tool for reliable cable monitoring for smart city infrastructures throughout the convergence with the various ubiquitous technologies. 


\section{Conflict of Interests}

The authors declare that there is no conflict of interests regarding the publication of this paper.

\section{Acknowledgments}

This study was supported by the Basic Science Research Program through the National Research Foundation (NRF) funded by the Ministry of Education, Science and Technology of Korea (no. 2010-0023404) and the Core Research Project of the Super Long Span Bridge R\&D Center (08 CTIPE01) funded by the Korean Ministry of Land, Transport and Maritime Affairs (MLTM). Their complete support is greatly appreciated.

\section{References}

[1] S. Park, J. -W. Kim, C. Lee, and S. -K. Park, "Impedance-based wireless debonding condition monitoring of cfrp laminated concrete structures," NDT \& E International, vol. 44, no. 2, pp. 232-238, 2011.

[2] C. Lee and S. Park, "Damage classification of pipelines under water flow operation using multi-mode actuated sensing technology," Smart Materials and Structures, vol. 20, no. 11, Article ID 115002, 9 pages, 2011.

[3] J. Min, S. Park, C. -B. Yun, C. -G. Lee, and C. Lee, "Impedancebased structural health monitoring incorporating neural network technique for identification of damage type and severity," Engineering Structures, vol. 39, pp. 210-220, 2012.

[4] C. Lee and S. Park, "De-bonding detection on a CFRP laminated concrete beam using self sensing-based multi-scale actuated sensing with statistical pattern recognition," Advances in Structural Engineering, vol. 15, no. 6, pp. 919-927, 2012.

[5] M. L. Wang, G. Wang, and Y. Zhao, Sensing Issues in Civil Structural Health Monitoring, Springer, Dordrecht, The Netherlands, 2005.

[6] M. L. Wang, Z. L. Chen, S. S. Koontz, and G. M. Lloyd, "Magnetoelastic permeability measurement for stress monitoring in steel tendons and cables," in Nondestructive Evaluation of Highways, Utilities, and Pipelines IV, vol. 3995 of Proceedings of the SPIE, pp. 492-500, March 2000.

[7] S. Sumitro, A. Jarosevic, and M. L. Wang, "Elasto-magnetic sensor utilization on steel cable stress measurement," in Proceedings of the 1st Fib Congress, pp. 79-86, Osaka, Japan, 2002.

[8] M. Göktepe, "Non-destructive crack detection by capturing local flux leakage field," Sensors and Actuators A: Physical, vol. 91, no. 1-2, pp. 70-72, 2001.

[9] C. Mandache, B. Shiari, and L. Clapham, "Defect separation considerations in magnetic flux leakage inspection," InsightNon-Destructive Testing and Condition Monitoring, vol. 47, no. 5, pp. 289-293, 2005.

[10] H. R. Weischedel, "The inspection of wire ropes in service: a critical review," Materials Evaluation, vol. 43, no. 13, pp. 15921605, 1985.

[11] J. Lee, J. Hwang, J. Jun, and S. Choi, "Nondestructive testing and crack evaluation of ferromagnetic material by using the linearly integrated hall sensor array," Journal of Mechanical Science and Technology, vol. 22, no. 12, pp. 2310-2317, 2008.

[12] O. L. Atherton, "Magnetic inspection is key to ensuring safe pipelines," Oil and Gas Journal, vol. 87, no. 32, pp. 52-61, 1989.
[13] H. R. Weischedel and C. R. Chaplin, "Inspection of wire ropes for offshore applications," Materials Evaluation, vol. 49, no. 3, pp. 362-367, 1991.

[14] K. Mandal, D. Dufour, T. W. Krause, and D. L. Atherton, "Investigations of magnetic flux leakage and magnetic Barkhausen noise signals from pipeline," Journal of Applied Physics, vol. 30, no. 6, pp. 962-973, 1997.

[15] K. K. Tandon, "MFL tool hardware for pipeline inspection," Materials Performance, vol. 36, no. 2, pp. 75-79, 1997.

[16] K. Mandal and D. L. Atherton, "A study of magnetic flux-leakage signals," Journal of Physics D, vol. 31, no. 22, pp. 3211-3217, 1998.

[17] J. E. Lenz, "Review of magnetic sensors," Proceedings of the IEEE, vol. 78, no. 6, pp. 973-989, 1990.

[18] E. Ramsden, Hall-Effect Sensors: Theory and Applications, Newnes, Oxford, UK, 2006.

[19] S. Coles, An Introduction to Statistical Modeling of Extreme Values, Springer, Berlin, Germany, 2001. 

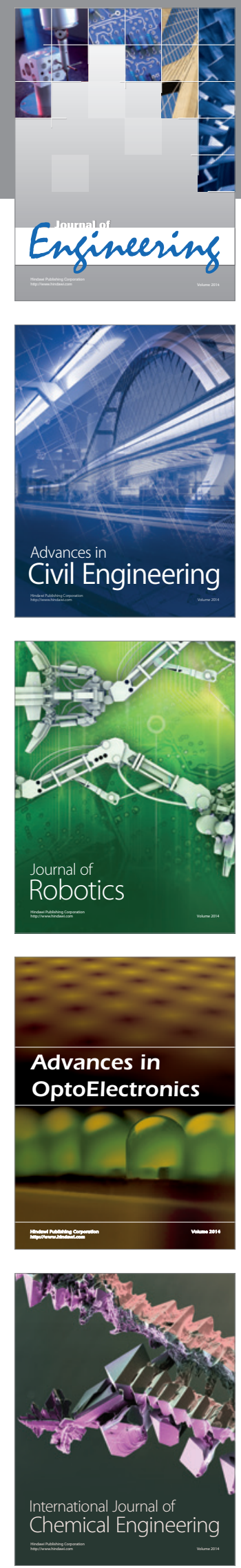

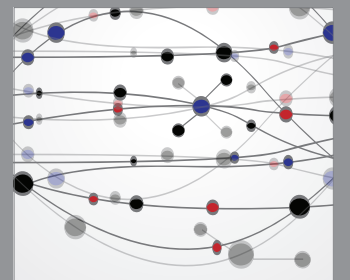

The Scientific World Journal
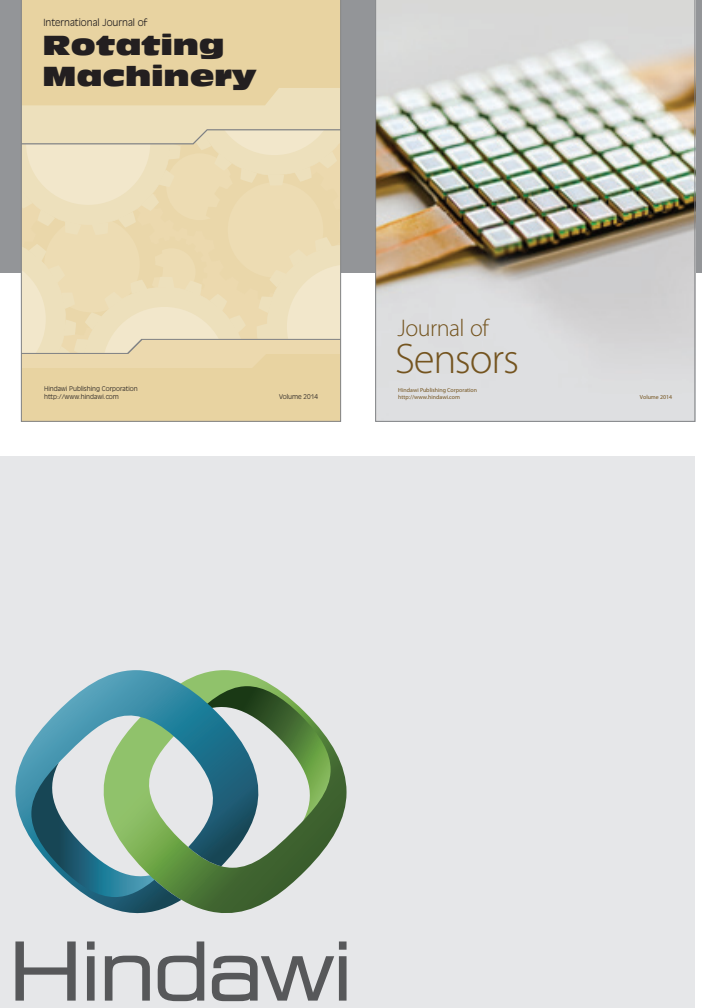

Submit your manuscripts at http://www.hindawi.com
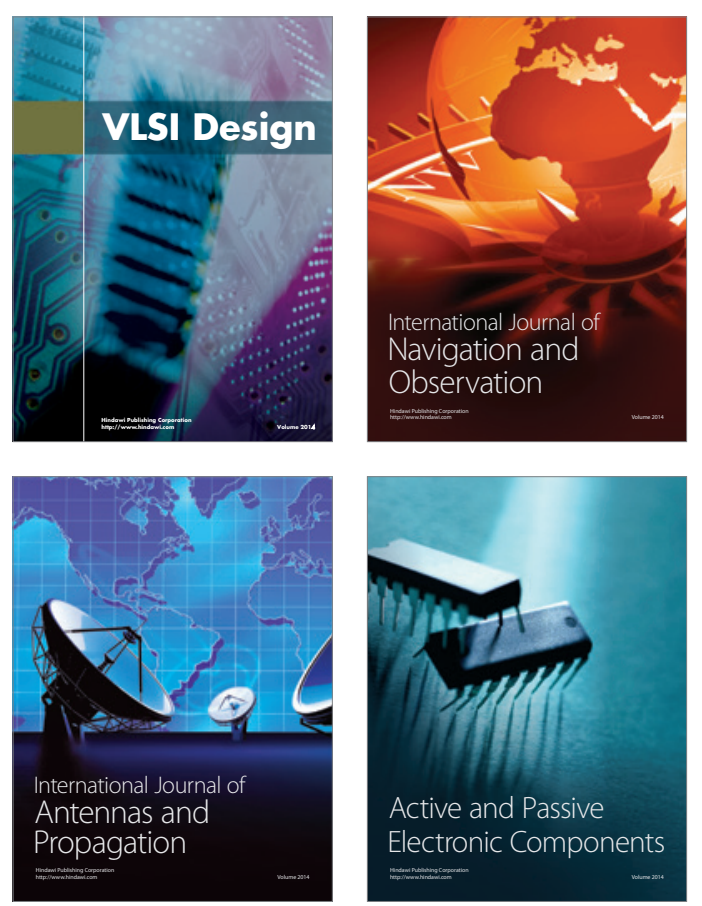
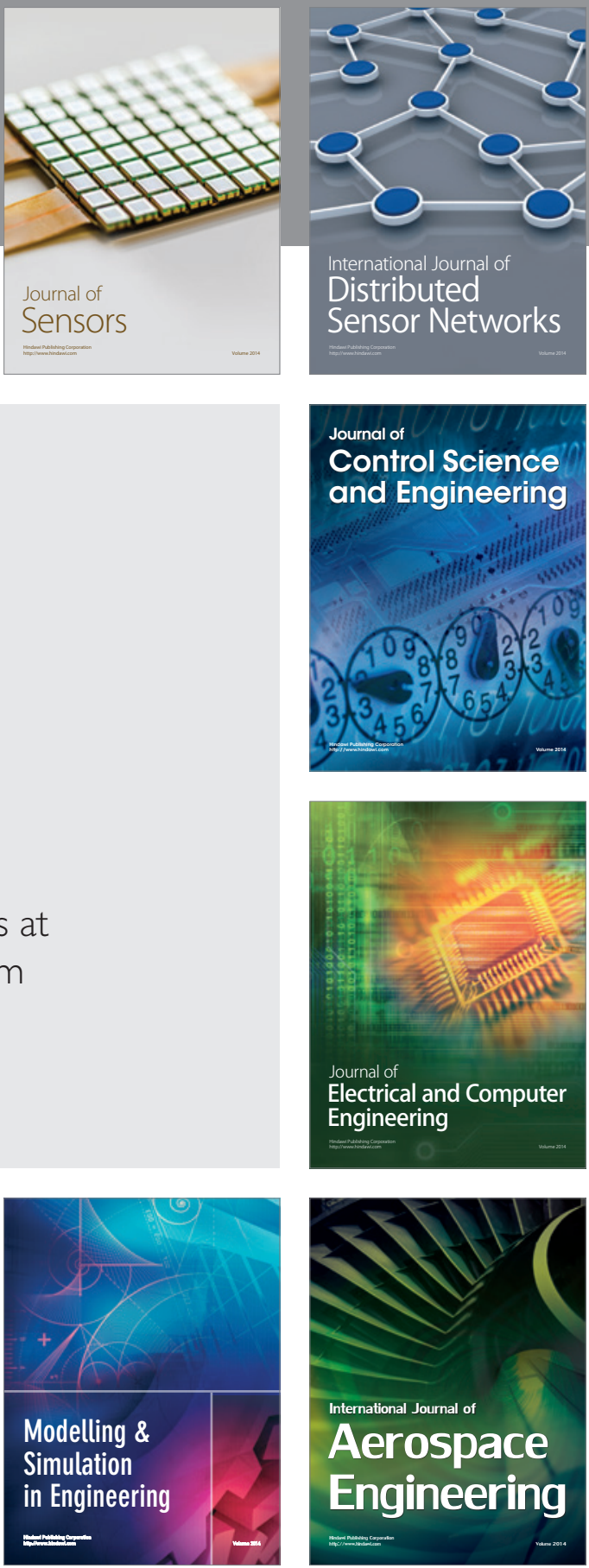

Journal of

Control Science

and Engineering
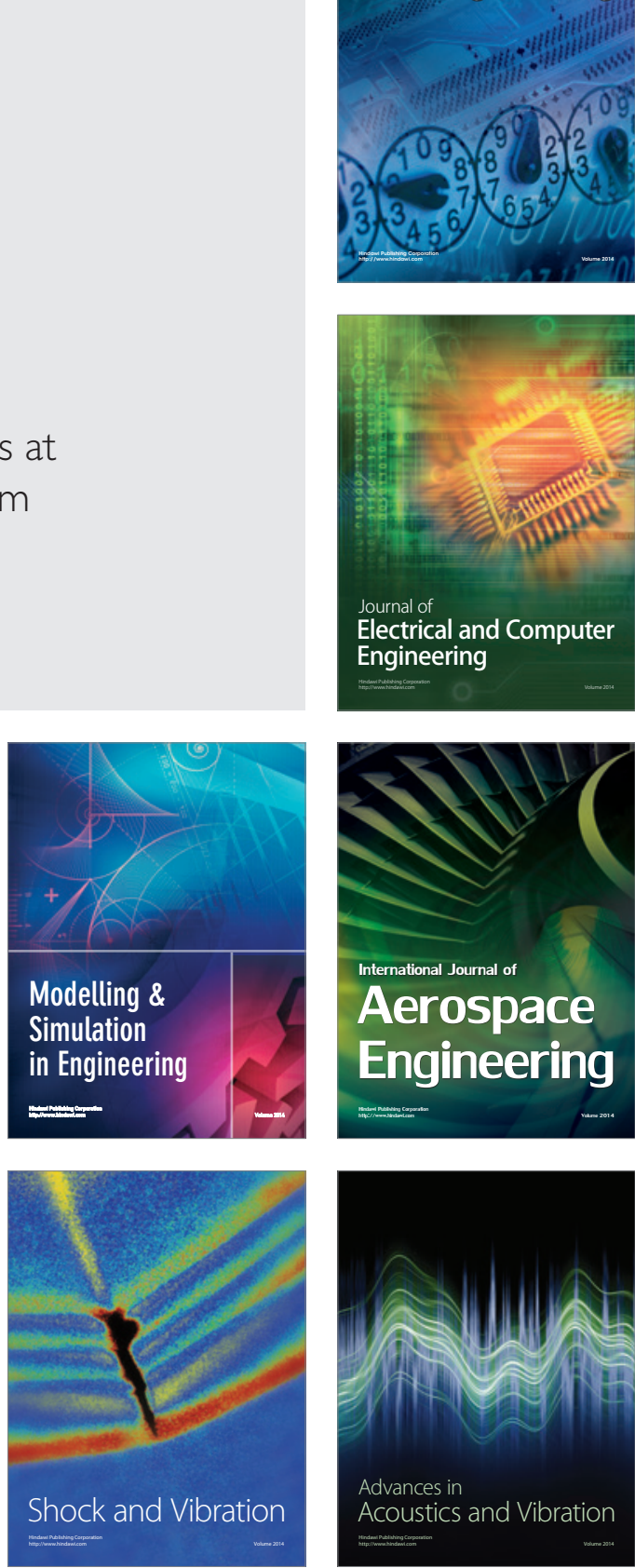